\title{
The Low- and Intermediate-Mass Stellar Population in the Small Magellanic Cloud: The Central Stars of Planetary Nebulae ${ }^{1}$
}

\author{
Eva Villaver ${ }^{2}$ \\ Space Telescope Science Institute, 3700 San Martin Drive, Baltimore, MD 21218, USA; \\ villaver@stsci.edu \\ Letizia Stanghellini ${ }^{3}$, and Richard A. Shaw \\ National Optical Astronomy Observatory, 950 N. Cherry Av., Tucson, AZ 85719, USA; \\ letizia@noao.edu,shaw@noao.edu
}

\begin{abstract}
We present a study on the central stars (CSs) of Planetary Nebulae (PNe) observed in the Small Magellanic Cloud (SMC) with the Space Telescope Imaging Spectrograph instrument on-board the HST. The stellar magnitudes have been measured using broad-band photometry, and Zanstra analysis of the nebulae provided the stellar temperatures. From the location of the CSs on the HR diagram, and by comparing the observed CSs with current models of stellar evolution, we infer the CSs masses. We examine closely the possibility of light contamination in the bandpass from an unrecognized stellar companion, and we establish strong constraints on the existence and nature of any binary companion. We find an average mass of $0.63 \mathrm{M}_{\odot}$, which is similar to the mass obtained for a sample of CSs in the LMC $\left(0.65 \mathrm{M}_{\odot}\right)$. However, the SMC and LMC CS mass distributions differ slightly, the SMC sample lacking an intermediate-mass stellar population $\left(0.65\right.$ to $\left.0.75 \mathrm{M}_{\odot}\right)$. We discuss the significance and possible reasons for the difference between the two mass distributions. In particular, we consider the differences in the star formation history between the clouds and the mass-loss rate dependence on metallicity.
\end{abstract}

Subject headings: Magellanic Clouds-planetary nebulae: general-stars: AGB and post-AGB-stars: evolution-stars: fundamental parameters- stars: mass-loss

\footnotetext{
${ }^{2}$ Affiliated with the Hubble Space Telescope Division of the European Space Agency

${ }^{3}$ On leave from INAF-Bologna Observatory

${ }^{1}$ Based on observations made with the NASA/ESA Hubble Space Telescope, obtained at the Space Telescope Science Institute, which is operated by the Association of Universities for Research in Astronomy, Inc., under NASA contract NAS 5-26555
} 


\section{INTRODUCTION}

Central Stars (CSs) of Planetary Nebulae (PNe) are the result of the evolution of lowand intermediate-mass stars $\left(1\right.$ to $\left.\sim 5 \mathrm{M}_{\odot}\right)$ that loose most of their stellar envelope through mass-loss towards the end of the Asymptotic Giant Branch (AGB) phase.

The CS mass depends mainly on the stellar mass during the Main-Sequence (MS) phase (hereafter, the initial mass), and on the mass-loss during the AGB. Neither the process responsible for the high mass-loss rates during the AGB phase nor its dependency on metallicity are well understood. In the commonly accepted scenario the mass-loss is thought to be driven mainly by a combination of two processes: dust formation by shock waves caused by the Mira-like stellar pulsation, and the acceleration of dust by radiation pressure (Wood 1979; Bowen 1988). The transfer of momentum from the dust to the gas ultimately drives the outflow.

The dust formation process depends on the chemical composition of the gas: the lower the metallicity, the smaller the amount of dust formed, and the lower the efficiency of the momentum transfer to the gas. Thus, low metallicity stars with dust-driven winds are expected to loose smaller amounts of matter (Winters et al. 2000). Willson (2000) has shown that mass-loss during the AGB phase can occur even in the absence of dust, but its efficiency is then much lower. In the case where the mass-loss occurs in the absence of dust, low metallicity stars are expected to have reduced mass-loss rates because they have a smaller radius for a given mass and luminosity (Willson, Bowen, \& Struck 1996; Willson 2000). Thus, everything, seems to conspire against mass-loss at low metallicity. As a consequence, a higher fraction of main sequence stars should reach the Chandrasekhar mass limit in the SMC than in the LMC (Umeda et al. 1999; Dominguez et al. 1999; Girardi et al. 2000), implying a higher rate of type II Supernovae explosions in the SMC than in the LMC, if we assume identical initial, low- and intermediate-mass stellar populations in the two Clouds.

Studies of CSs in the Galaxy are hampered by the poor knowledge of their distances, a problem that can be overcome by observing PNe in the Magellanic Clouds (LMC and SMC) (Stanghellini 2000). The high spatial resolution capabilities of the Hubble Space Telescope (HST) is perfectly matched to resolve the Magellanic Cloud PNe, and to directly observe their CSs. Furthermore, LMC and SMC CSs are affected by low field reddening. Since the metallicity of the LMC and SMC are on average half and quarter that of the solar mix (Russell \& Bessell 1989; Russell \& Dopita 1990), studying samples of CSs in both Clouds allows one to directly probe stellar evolution and mass-loss with different metallicities. In this paper, we determine the mass of a sample of CSs in the SMC. The aim is to compare them with a similarly-selected sample of LMC CSs (Villaver, Stanghellini, \& Shaw 2003; 
hereafter Paper I) to explore the effect of a different metallicity on the CS mass distribution. The goal of this series of papers is to determine accurate CSs masses of a large number of extragalactic PNe, and to ultimately relate the final mass to the initial mass of these sub-Chandrasekhar mass stars.

We present Space Telescope Imaging Spectrograph (STIS) broad-band imaging of 27 targets in the SMC. In $\S 2$ we describe the observations. In $\S 3$ we present the methodology and the results of the stellar photometry, the Zanstra temperature derivation, the bolometric correction, and the derivation of the stellar luminosities; in $\S 4$ we discuss the correlations and the statistics of the measured stellar parameters; finally, in $\S 5$ we discuss and summarize our findings.

\section{OBSERVATIONS}

The observations of the 27 targets presented in this paper are from the HST SNAPSHOT program 8663. The observations were made in direct imaging (50CCD) and in slitless mode with the G430M and G750M gratings of STIS. The observing log, observing configuration, target selection, acquisition, and basic calibrations are described in Stanghellini et al. (2003), as well as the morphological classification of the nebulae, the line intensities, and other nebular diagnostics obtained from the slitless spectra. In this paper we present the photometric analysis performed on the STIS broad-band images (50CCD). The 50CCD has sensitivity from $\sim 2,000$ to $10,300 \AA$ and a spatial scale of $0^{\prime \prime} .0507 \mathrm{pix}^{-1}$. The FWHM is close to 2 pixels at the central wavelength of the 50CCD $5850 \AA$ (Leitherer et al. 2001). The observations were made with the CCD detector using a gain of $1 \mathrm{e}^{-}$per analog-to-digital converter unit. All the exposures were split into two equal components to facilitate cosmic-ray removal. In Table 1 we give in column (1) the PN name (we favor the SMP nomenclature, when available); column (2) the total integration time, and in column (3) we note whether the CS has been detected in the image. 


\section{ANALYSIS}

\subsection{Stellar Photometry}

The technique to measure the CS photometry has already been described in detail in Paper I. To summarize it, we have applied aperture photometry using the IRAF ${ }^{2}$ phot task. We have considered a stellar aperture with a radius of 2 pixels to measure the flux of the CS. The contribution from the nebula in the stellar aperture has been subtracted by estimating the median nebular flux in an annulus with a width of 2 pixels adjacent to the stellar aperture. At the distance of the SMC, the HST spatial resolution allowed us to resolve the nebula and separate the nebular and stellar emission. The variation of the nebular flux within the annulus, is reflected in the standard deviation and is propagated in the errors of the measured magnitudes. The fraction of the stellar point spread function (PSF) that falls out of the stellar aperture has been corrected using an aperture correction determined from the curve of encircled energy derived by Brown et al. (2002) for stars near the field center.

There are four unresolved objects (i.e. objects that have nebular extension similar to the stellar PSF) in our sample, J 23, SMP 1, SMP 18, and SMP 20. In these cases, the nebular morphology is not available, however, we can still detect the CS and measure its magnitude in all of the PNe except SMP 18. Among the other PNe, J 18, SMP 6, and SMP 24 are the most compact, yet spatially resolved. As a consequence, the nebular contribution to the continuum that we subtract to obtain the stellar magnitude is high and it has a small standard deviation which is reflected in the reduced uncertainties in the measured magnitude. Thus it should be noted that the magnitude errors given for J 18, SMP 6 and SMP 24 are probably underestimated.

When the CS is detected at a very low level above the nebular emission, or the nebular emission decreases very sharply with the distance from the star we can considerably reduce the systematic photometric errors by subtracting a nebular image before performing the photometry. The method has been described in detail in Paper I. The nebular image is built by stacking the monochromatic images, $\mathrm{H} \beta$, [O III] 4959, $5007 \AA$, $\mathrm{H} \alpha$, and [N II] 6548 and $6584 \AA$ lines, obtained from the slitless STIS spectra. In the case where the CS is not detected in the 50CCD images, we have estimated a lower limit to the CS magnitude by performing aperture photometry at the geometrical center of the nebulae.

\footnotetext{
${ }^{2}$ IRAF is distributed by the National Optical Astronomy Observatory, which is operated by the Association of Universities for Research in Astronomy, Inc., under cooperative agreement with the National Science Foundation.
} 
The zero-point calibration to magnitudes in the $\mathrm{STMAG}^{3}$ system has been taken from Brown et al. (2002). As in Paper I, we have neglected the STIS charge transfer efficiency (CTE), since it has been shown to be below 0.01 dex (Rejkuba et al. 2000) for stars near the field center. The image distortions can also be neglected, since they are known to be negligible in this observing mode.

The magnitudes have been corrected for extinction using the amount of nebular extinction derived from the Balmer decrement, and the relation $\mathrm{c}=1.41 \mathrm{E}_{B-V}$ (Seaton 1979). The logarithmic extinction constant at $\mathrm{H} \beta$, c, has been taken from Stanghellini et al. (2003) except for J 18 (Jacoby \& Kaler 1993), J 23, and J 27 (Boroson \& Liebert 1989), MG 13 (Leisy \& Dennefeld 2003), and SMP 20 (Leisy \& Dennefeld 1996) for which we have used the values given in the literature since no G430M exposures were available. We have used the interstellar extinction law of Savage \& Mathis (1979), and assumed that $\mathrm{R}_{V}=3.1$ in order to derive the extinction, because in our wavelength range, the SMC extinction law is very similar to the Galactic extinction law (Howarth 1983).

We have modeled the transformation from instrumental magnitudes in the STMAG system to standard V-band magnitudes in the photometric Johnson-Cousins UBVI system with IRAF/STSDAS ${ }^{4}$ task synphot. The median of the V-50CCD colors obtained for blackbodies between 30,000 and $300,000 \mathrm{~K}$ with $\mathrm{E}_{B-V}$ values appropriate for each source has been used to transform the STMAGs into V-band magnitudes (see Paper I for details). The uncertainty in the transformation has been added in quadrature to the error in the magnitude.

In Table 2, we have summarized the results of the photometry. Column (1) gives the PN name; columns (2) and (3) give the STMAG and standard V magnitudes respectively, or their lower limits, as well as the associated errors. The errors quoted in this Table include several sources: the random error (e.g., the photon and read-out noise), the systematic errors (from the CS fluxes, the sky subtraction, etc), and the errors in the calibration. The observations of J 4 and SMP 18 were saturated, and are noted in the Table. The magnitudes derived from saturated data have not been included in the analysis, the mass calculation and the plots. The extinction constants used to determine the color excesses needed to correct for extinction are listed in column (4).

\footnotetext{
${ }^{3}$ The STMAG is the Space Telescope magnitude system, based on a spectrum with constant flux per unit wavelength.

${ }^{4}$ STSDAS is a product of the Space Telescope Science Institute, which is operated by AURA for NASA.
} 


\subsection{Effective Temperatures, Bolometric Corrections, and Stellar Luminosities}

Of the different methods available in the literature to determine the stellar temperature (e.g. Stoy's method; Stoy 1933) we have adopted the Zanstra method (Zanstra 1931; Harman \& Seaton 1966; Kaler 1983). The Zanstra method relates the total ionizing flux of the star (hereafter assumed to have a blackbody spectrum) to the flux of a recombination line (of hydrogen or helium) and to the stellar continuum flux in the $\mathrm{V}$ band. The implicit assumption behind the Zanstra method is that all the photons above the Lyman limit of $\mathrm{H}_{\text {or }} \mathrm{He}^{+}$are absorbed within the nebula, and that each recombination results in a Balmer photon. When the He II $4686 \AA$ line flux is available, we estimate two values for the stellar temperature, which are based on the hydrogen and the helium recombination lines.

The data needed for the temperature calculation ( $\mathrm{H} \beta$ fluxes, nebular radii, and extinction constants) have been taken from Stanghellini et al. (2003) when available. The H $\beta$ fluxes and extinction constants for J 18, J23, and J 27 have been taken from the literature (Jacoby \& Kaler 1993; Boroson \& Liebert 1989). In the case of MG 13 the extinction constant has been taken from Leisy \& Dennefeld (2003) and the $\mathrm{H} \beta$ flux has been computed from the $\mathrm{H} \alpha$ flux given in Stanghellini et al. (2003) by assuming case B recombination from Osterbrock (1989), and a nebular gas temperature of 10,000 K. The He II $4686 \AA$ line fluxes have been taken from Boroson \& Liebert (1989), Leisy \& Dennefeld (1996, 2003), Dopita \& Meatheringham (1991a,b), Vassiliadis et al. (1992), Monk, Barlow, \& Clegg (1988), and Jacoby \& Kaler (1993). In order to assure the best results we have been very conservative with the errors in the fluxes quoted by the references. In Table 2 column (4) we have listed the 4686 He II flux line intensities relative to $\mathrm{H} \beta=100$, not corrected for extinction (and their errors), and in column (5) we list the reference code (see the legend in the note to the Table) for the He II fluxes.

In order to compute the bolometric correction (BC), we have used the relation between BC and $\mathrm{T}_{\text {eff }}$ given by Vacca, Garmany, \& Shull (1996). When available, the He II Zanstra temperature has been used to determine the BC (unless it has been derived from upper limits to the $4686 \mathrm{He}$ II flux), since they are the most reliable, otherwise the H I Zanstra $\mathrm{T}_{\text {eff }}$ has been used. The CSs luminosities have been computed by adopting a distance to the SMC of 58.29 Kpc (Westerlund 1997), and an absolute solar bolometric magnitude of $\mathrm{M}_{b o l, \odot}=4.75 \mathrm{mag}$ (Allen 1976).

The SMC has an intrinsic line of sight depth between 4 and $16 \mathrm{kpc}$ (Hatzidimitriou \& Hawkins 1989; Hatzidimitriou, Hawkins, \& Gyldenkerne 1989; Gardiner \& Hawkins 1991; Gardiner \& Hatzidimitriou 1992), depending on the portion of the SMC considered. In a more recent paper, Crowl et al. (2001) obtained a line of sight depth between 6 and $12 \mathrm{kpc}$ by using $12 \mathrm{SMC}$ clusters. The positions of the CSs detected in our sample span a range of 
$400 \mathrm{pc}$ in right ascension and $2 \mathrm{kpc}$ in declination with respect to the optical center of the SMC. From the dispersion in the distance to the SMC derived by Crowl et al. (2001) from the analysis of SMC clusters positions we have estimated an average line of sight depth of $5 \mathrm{kpc}$ for the CSs in our sample. The distance uncertainty introduced by this depth in the $\mathrm{SMC}$ will systematically increase the $\log \mathrm{L}$ errors by 0.02 . We therefore conclude that the error in the luminosity due to the 3-dimensional structure of the SMC is negligible.

In Table 3 we give in column (1) the PN name; in columns (2) and (3) we give the effective temperature (in units of $10^{3} \mathrm{~K}$ ) derived from the Zanstra method for the helium and hydrogen recombination lines respectively. Column (4) gives the absolute visual magnitude; column (5) gives the stellar luminosity. The BC and its error, computed by propagating the error in the temperature is given in column (6). The morphological classification in column (7) is from Stanghellini et al. (2003). Column (8) contains comments relevant to the magnitude measurement.

\section{RESULTS}

\subsection{Effective Temperatures}

In Figure 1 we compare the ratio of the stellar effective temperature derived from the He II $4686 \AA\left[\mathrm{T}_{Z}(\mathrm{He}\right.$ II $\left.)\right]$ and the $\mathrm{H} \beta\left[\mathrm{T}_{Z}(\mathrm{H})\right]$ recombination lines (the Zanstra ratio) versus the He II Zanstra temperature. It should be noted that, although we have computed a $\mathrm{T}_{Z}$ (He II) upper limit using the upper limit He II $4686 \AA$ flux for some of the objects (see Table 3), we do not include them in the plot, or in the calculations. The Zanstra ratio approaches unity as $\mathrm{T}_{Z}$ (He II) increases, which is explained as an effect of the optical thickness of the nebula to the hydrogen ionizing radiation (Kaler \& Jacoby 1989; Gruenwald \& Viegas 2000). A similar result was also found for the LMC CSs in paper I.

\subsection{Luminosity-Nebular Radius and Surface Brightness Relations}

In Figure 2 we explore the relation between the CS luminosities derived in this paper and the PN photometric radius (in pc) from Stanghellini et al. (2003). The CS and the nebula are interdependent systems, that is, the evolution of the nebula is governed by the CS energetics, mainly through the stellar wind and the ionizing radiation field, which are both a function of the CS mass and its evolutionary status. Numerical simulations are required to understand how the evolution of the CS luminosity relates to the nebular radius. Such models are currently unavailable for the Magellanic Cloud PNe, however, as in Paper I, we 
can obtain a qualitative indication of how the evolution of nebular radius and CS luminosity relate to each other by using the models for Galactic PNe of Villaver et al. (2002ab). In the Figure, the symbols represents different morphologies (as in Fig. 1) and each line is labeled with the initial stellar mass used in the simulation.

In Figure 3 we explore the relation between the stellar luminosity and the nebular surface brightness in the $\mathrm{H} \beta$ emission line $\left(\mathrm{SB}_{H \beta}\right.$, defined as the integrated line flux divided by the nebular area $\pi \mathrm{R}_{\text {phot }}^{2}$; where $\mathrm{R}_{\text {phot }}$ is the photometric radius). As in Fig. 1 the symbols represent the different morphological types. We find that low SB objects are always located towards the position of the low luminosity CSs. The relation is very similar to the one found for the LMC CSs in Paper I. The 3 objects located outside the main trend are (from the top left to the right) SMP 25, MG 8 and MG 13. This plot is a representation of the related fading of the CSs and the nebulae, as seen with snapshots of the observed populations.

\subsection{Stellar Distribution on the Log L-Log T Plane, and the CSs Masses}

In Figure 4 we show the distribution of the detected CSs in the Log L-Log T plane. The evolutionary tracks for the SMC metallicity (Vassiliadis \& Wood 1994) encompass the observations well. The CS masses listed in Table 4 have been derived by comparing their location on the HR plane with the H-burning tracks of Vassiliadis \& Wood (1994), except in the case of J 18, whose mass was determined from its position with respect to a He-burning track. Note that the CS temperatures and luminosities (and therefore the masses) have been computed within the assumption of a non-binary CS. The viability of the assumption will be addressed in the following sub-section and in the discussion ( $\$ 5)$. We have not derived masses for the two stars that lie below the evolutionary tracks (SMP 11 and SMP 12).

In the cases where the helium flux at $\lambda 4686$ was not available (SMP 1, SMP 6, SMP 8, SMP 17, SMP 20, SMP 24, and SMP 27), we have used the $\mathrm{T}_{Z}(\mathrm{H})$ to locate the CSs on the HR diagram. $\mathrm{T}_{Z}(\mathrm{H})$ underestimates the temperature of the CSs hosted by optically thin PNe but it is accurate for optically thick objects. Although the He II 4686 fluxes of SMP 1, SMP 6, SMP 17, and SMP 20 are only upper limits, and are a factor of 100 smaller than the $\mathrm{H} \beta$ flux, we still determine $\mathrm{T}_{Z}$ (He II). However, as the uncertainties are expected to be very high, we have used $\mathrm{T}_{Z}(\mathrm{H})$ to derive the masses. The difference in the CS masses derived using $\mathrm{T}_{Z}\left(\right.$ He II) or $\mathrm{T}_{Z}(\mathrm{H})$ is significant for SMP 1 and SMP 6, however, it is almost zero for SMP 24, and SMP 20. 


\subsection{Constraints on a Potential Stellar Companion}

In the above, we implicitly assumed that all the light measured in the stellar aperture is actually arising from the exciting star. We do not have color information, and our magnitudes are measured through a broad-band filter. If the light in the aperture is contaminated by a companion star (that is if the CS is embedded in a binary system) then the actual brightness of the PN progenitor in the passband would be fainter, which would mean that the true CS temperature and luminosity would be different from what was derived assuming no companion. In this subsection we quantify the effects of hypothetical stellar companions, subject to the strong constraint of a well determined distance, and examine the consequences on the inferred temperature, luminosity, and mass of the PN progenitors.

A well determined distance modulus to the SMC allows strong constraints to be placed on the luminosity class of any binary companions to the CSs. Specifically, we can easily rule out supergiants (luminosity class I) as companions because such stars would be brighter in the STIS/50CCD bandpass than any CS in our sample. SdO Sub-dwarf companions are extremely unlikely because they evolve so quickly that they produce significant light in the $\mathrm{V}$ bandpasss (compared to the CSs) for only $\sim 10^{5} \mathrm{yr}$ after evolving of the AGB phase. Giant companions (luminosity class III) do produce the appropriate flux in our bandpass, so such companions cannot be ruled out. However, we believe it is statistically unlikely that many CSs in our small sample have Giant companions, given the brevity of this phase of evolution (Renzini \& Buzzoni 1986). Yungelson, Tutukov, \& Livio (1993) modeled a population of all binary stars and estimated the birthrates of binary CSs of different types. By this estimate, the birthrate of a binary CS formed by a CO white dwarf with a Giant companion is only $0.044 \%$ that of a CS formed by a CO white dwarf with a main-sequence companion. While there are a few PNs in the Galaxy with CS companions that are known to be Giants (e.g. A 35; Jacobi 1981), we believe such cases are intrinsically rare and are unlikely to affect the conclusions of this paper.

The combination of spectral type and luminosity class allows us to set strong constraints on the possibility of MS companions in our sample of CSs. We have recalculated the $V$ magnitudes of the CSs in Table 4 (those with derived masses) by assuming different MS binary companion contributions to the light in the bandpass. By using synphot we compute the instrumental response to an input spectrum renormalized to the measured count rate. We have considered a MS companion star that generates $50 \%, 90 \%$, and $95 \%$ of the measured V-band flux (by using the synthetic spectral atlas from Bruzual). Since the distance modulus for the SMC is well known, the flux limits severely limit the spectral type of the companion (Cox 2000). Basically, we adjust the $V$ magnitude of the model to match the observed counts

for our observing mode. Note that then the CS $V$ magnitude changes to get the measured 
count rate.

With this revised CS $V$ magnitude we recalculate the effective temperatures, BCs, and CS luminosities following the procedure described in $§ 3.2$. CS masses have been derived as well based on the new position of the CSs in the HR diagram (as in §4.3).

In Table 5 we give the recalculated CS parameters under different assumptions about the relative contribution from a hypothetical MS companion star. Column (1) lists the PN name, columns (2), (3), (4), and (5) we give the assumed percentage of the companion to the count-rate, the $V$ magnitude that the companion would have with the assumed contribution to the flux, the spectral type that would correspond to this $V$ at the distance of the SMC and the MS mass of the hypothetical companion from Cox (2000) respectively. Columns (6), (7), (8), (9), and (10) lists for the CS, $V$, the effective temperature, the stellar luminosity (in Log), the CS mass and its infered MS mass respectively. The latter has been derived from the initial- to final-mass relation given in Vassiliadis \& Wood (1994) for CSs in the SMC. Obviously, in those cases where the light is dominated by the true CS our mass determinations in Table 4 will not be affected. Moreover, an arbitrarily small contributions from the companion it is not worth considering because then our estimate for the CS mass does not change meaningfully.

Comparison between columns (5) and (10) tell us whether a binary MS companion is physically reasonable. When the MS mass of the companion is larger than the initial mass derived for the CS we fall into an evolutionary contradiction: a less massive star cannot have evolved into a PN before its more massive MS companion. Moreover, the difference in mass between the two components of the system has to be large enough to allow one of them to evolve into the PN domain while the other is still in the MS. This is correct within the assumption that no significant mass-transfer has occured between the CS progenitor and the postulated companion. PN formation in a close binary system depends in detail upon the masses and structure of the component stars at the time the (initially) more massive companion evolves off of the MS (Iben 1991). A detailed interpretation in this context is beyond the scope of this paper. From Table 5, by assuming that it will lead to an evolutionary contradiction, we can safely rule out the possibility of light contamination in all the objects, except in SMP 9, SMP 14, and SMP 20. These three PNe deserve further investigation.

The ionization status of the nebula is a direct reflection of the effective temperature of the CS. Zanstra analysis gives larger effective temperatures when the CS magnitude value increases for a given nebular flux. For SMP 9, SMP 14, and SMP 20 we have checked for consistency between what would be the CS effective temperatures admiting the possibility of a binary and the nebular photoionizacion spectra. We have computed the excitation class (EC) of the nebula based on the definition of Dopita \& Meatheringham (1990) and 
the nebular fluxes in the literature (same references as in Table 2). We have used the relation between EC and effective temperature of the CS given by Dopita \& Meatheringham (1991b). This relation is purely based on photoionization models and spectroscopic data for the nebula. They are, then, safe from the photometric contamination that might be affecting SMP 9, SMP 14, and SMP 20.

For SMP 14 and SMP 20 the measured light in the band-pass would have to be completely dominated by the companion (above $90 \%$, see Table 5) which would lead to effective temperatures above 200,000 K. The ECs computed for the nebula are 5 for SMP 14 , and 0 for SMP 20 corresponding to effective temperatures of 100,000 and 40,000 K respectively. Light contamination from a binary companion in our photometry would imply CS temperatures are inconsistent with the ionization status of these nebula. To stress this point note also that the NeV $3426 \AA$ line have not been detected in either of these PNs. This line being an additional indicator of EC above 8 ( $\left.\mathrm{T}_{\text {eff }} \geq \sim 180,000 \mathrm{~K}\right)$ (Pottasch 1984).

A MS companion for SMP 9 emiting more than $65 \%$ of the flux will move the CS outside the PN tracks in the HR diagram. A CS temperature consistent with the presence of a MS K0 or G8 companion (which will have to contribute less than $65 \%$ of the measured flux) will have to be above $230,000 \mathrm{~K}$ to affect meaningfully the derived core mass. This temperature correspond to an EC above 8 for the nebula which is inconsistent with the EC derived from the nebular spectra, 5. The NeV $3426 \AA$ line have not been detected in this nebula. We can not exclude, however, the presence of a MS star contributing less than $50 \%$ of the measured light. Thus it is possible that in SMP 9 the detected CS includes light from a companion star. Note, however, that light contamination in this case would not meaningfully affect the mass derived for the CS.

We conclude that we are likely not observing a companion star in any of the CSs in our sample, apart from the exceptions noted above, either because the flux from such companions would not be consistent with what is observed in our bandpass, or because the $\mathrm{L}, \mathrm{T}$, or the mass implied for a fainter CS would either be evolutionarily unreasonable or would be inconsistent with the excitation of the nebula, or because the likelihood of plausible companions is fairly small. It is difficult to apply the knowledge of the incidence of binary CSs in Galactic PNe, owing in part to the difficulty of demonstrating a large and volumecomplete sample in the Galaxy (Pottasch 1996). If we assume that the $10 \%$ fraction reported by (Bond 2000) for Galactic PNe also holds for the SMC, then we would expect two of the 21 positive detections in our sample would be in a close binary system. We have determined CS masses for only 14 objects, so it might be that one or two of the CSs actually has a close binary companion, which is consistent with what we found in the analysis above. Additional spectroscopy of the CSs in our sample would resolve the question. 


\subsection{Comparison of the SMC and LMC CSs masses}

The sample of SMC CSs analyzed in this paper and the sample of LMC CSs observed with STIS in Paper I can be usefully compared for statistical studies. The original samples were selected in a similar fashion, and were observed with the same HST instrument and observing configuration. The samples are of comparable size, and a similar fraction of CSs were detected within the observed PNe (67\% and $74 \%$ for the SMC and LMC respectively).

In Table 5 we give the basic statistics for the CSs masses, the effective temperatures, and stellar luminosities of the SMC and LMC CSs. In column (1) we give the galaxy and, in parenthesis, the sample size. Columns (2), and (3) show the mean and the median of the mass distributions respectively. Columns (4), and (5) and (6), and (7) give similar statistics for the temperature and luminosities distributions (in logarithmic scale). The numbers given in Table 5 also include SMP 1 and SMP 6, despite their masses having large errors (see the discussion in §5.3). If we exclude SMP 1 and SMP 6 from the SMC sample, the calculation of the mean mass only changes by $1 \%$ percent, and so does not affect our conclusions.

We find that the effective temperatures and luminosities (in logarithmic scales) of the two CS samples are consistent with Gaussian distributions. The CS masses, however, do not seem to be normally distributed. It is important to note that our samples of CS masses have been determined free from distance uncertainties, thus their non-Gaussian distribution is noteworthy. In the theoretical PN luminosity function and other PN applications, the CS mass distribution is generally assumed to be Gaussian. We will examine the consequences of a non-Gaussian CS mass distribution in a future paper.

In Figure 5 we show the normalized histogram of the mass distribution obtained for the SMC and the LMC samples (where the entire histograms have been normalized to have a maximum value equal to 1). From Fig. 5 we can see that the SMC hosts a larger fraction of low-mass CSs than the LMC. The SMC sample lacks a population of stars between 0.65 and $0.75 \mathrm{M}_{\odot}$ (see Table 4 ). We have tried to determine whether the mass distributions of the LMC and SMC CSs are significantly different by applying the Kolmogorov-Smirnov test (KS-test) (Chakravarti, Laha, \& Roy 1967). The number of CSs masses is 14 and 16 for the SMC and LMC data respectively, and therefore the effective number of data points (defined as the product of the number of data of each sample divided by its sum) is 7.4, the KS-test is appropriate for an effective number of data points greater than 4. The maximum difference, $\mathrm{D}$, between the cumulative distribution of the two datasets is 0.41 with a corresponding probability, $\mathrm{P}$, that the two distributions are the same of 0.12 . $\mathrm{P}=1$ indicates that two distributions are identical. Figure 5 and the result of the KS-test for the mass distributions, suggests that the mass distribution of CSs in the LMC and SMC are different. 
In Figure 6 we have plotted the SMC and the LMC CSs on the HR diagram, with different symbols. It is apparent from Fig. 6 (and from Table 5) that the LMC CSs are hotter, and the stellar luminosities have a larger dispersion. We have run a generalization of the KS test (Fasano \& Franceschini 1987; Peacock 1983) to test if the 2-dimensional distribution in the $\log \mathrm{L}-\log \mathrm{T}$ of the LMC and the SMC CSs differs. We found D $=0.34$ and $\mathrm{P}=0.37$, which is inconclusive in stating that the locations in the HR diagram of the LMC and SMC CSs are drawn from different distributions.

\section{SUMMARY AND DISCUSSION}

We have obtained the physical parameters of 14 CSs in the SMC. We have explored possible correlations between the CS (luminosity, effective temperature, and mass) and the nebula (photometric radius and SB). We find a similar pattern of evolution between the nebula and the star as found for the LMC CSs in Paper I. We confirm that the stellar luminosity evolves with nebular radius, as predicted by hydrodynamic models (Villaver, Manchado, \& García-Segura 2002b). We find a relation between the stellar luminosity and the PN surface brightness in the SMC CSs, as was found in the LMC sample. We do not find any significant relation between morphology and CS mass in the SMC sample, although it should be noted that there are very few asymmetric PNe with detected CSs in the SMC, and generally the undetected CSs will be the more massive.

Given the additional restriction that a known distance imposes to the measured flux we have explored the possibility that light from a stellar companion is contaminating the photometric measurements. After a detailed analysis we were able to establish strong constraints on the existence and types of companions that would be consistent with the observed stellar and nebular fluxes. We showed that no CS could have a Supergiant companion, and we have argued that sdO sub-dwarf and Giant companions are very unlikely. We also showed that MS companions are are not consistent with the data and the implied evolutionary state of the PN progenitor, except for the case of SMP 9 where the feasible MS companion would

not meaningfully affect the mass determined for the PN progenitor. It is important to note that our analysis does not exclude the possibility of a binary companion to the PN progenitor: we only limit or exclude the possibility of a companion as the source of the flux in the STIS/50CCD bandpass.

We find that if the CS is evolving in a binary system the mass determined within the assumption of a single CS will be an underestimation of the CS mass if the CS is on a cooling track on the HR diagram. A lower (or the same) mass will be determined if the CS is on the constant luminosity portion of the track. A hotter CS results if we allow flux contamination 
from a binary CS in the filter. While we cannot entirely rule out this possibility with the data in hand, we have been allocated HST Cycle 13 orbits that will allow us to increase the sample size and explore further its statistical significance.

We find a difference in the mass distribution of the SMC and the LMC CSs: the SMC has a narrower CS mass distribution than the LMC. Our findings are hampered by (1) the low number of SMC objects with helium Zanstra temperature determinations; (2) the high number of LMC CSs masses determined from helium-burning tracks (resulting in slightly lower masses), and (3) the overall small number of objects in the LMC and SMC samples. We should note that the bias of point (2) above strengthens the conclusion that the SMC has a narrower distribution of CS masses than the LMC.

There are indications that the SMC PN population hosts slightly lower mass CSs compared to the LMC. If the initial mass distributions in the galaxies in the 1-5 $\mathrm{M}_{\odot}$ range were the same, we would expect to find the opposite, that is, higher final masses in the SMC compared to in the LMC, a consequence of the reduced mass-loss rate expected in a lower metallicity environment. Since there is no evidence of a dependency of the IMF with metallicity (Salpeter 1955; Scalo 1998), we look to the history of star formation in the Magellanic Clouds to check whether we can expect differences in the final mass distributions.

It seems well established that the star formation history of the LMC and SMC are distinct (Olszewski, Suntzeff, \& Mateo 1996). The LMC experienced an episode of star formation $\sim 3-5$ Gyr ago (Bertelli et al. 1992), while the SMC seems to have been forming stars at a constant rate during the last 2-12 Gyr (Dolphin et al. 2001). The stars with ages between $7.8 \times 10^{8}$ and $2.7 \times 10^{8}$ yr are abundant in the LMC but are missing in the SMC. This latter population may correspond to a burst of star formation in the LMC. Thus, if the IMF is the same, then the star formation history differences may account for the differences in the observed mass distribution.

Dopita, Lawrence, Ford, \& Webster (1985) found that the kinematics of the SMC PNe is that of a spheroidal population without rotation. A study of Carbon stars, another intermediate-age stellar population, affords a similar scenario (Hardy, Suntzeff, \& Azzopardi 1989; Hatzidimitriou et al. 1997), in agreement with the SMC PN population being kinematically old.

If we consider the initial-to-final mass relation and the evolutionary timescales given by Vassiliadis \& Wood (1994), we find that our sample of CSs has an initial mass distribution that peaks at $\sim 1.5 \mathrm{M}_{\odot}$, which would translate to an age of $\sim 3$ Gyr. SMP 25 and MG 8 are the most massive SMC CSs in our sample. Their inferred initial masses (4.2 and 4.9 $\mathrm{M}_{\odot}$ respectively) sets a lower limit to the age of the SMC PN population, $\sim 10^{8} \mathrm{yr}$. These 
two PNe are located in the same region of the SMC, the northeast region, where Dopita, Lawrence, Ford, \& Webster (1985) found the kinematically younger PN population to be

concentrated. Crowl et al. (2001) found also that the eastern region of the SMC, which faces the LMC, contains younger and more metal rich clusters. Our estimated ages are then consistent with other studies of low- and intermediate-mass populations.

We cannot definitively claim that the difference found in the mass distribution between the two Clouds is caused by differences in the star formation histories, however we argue that it can explain it. A metallicity dependency on the mass-loss rate alone, is not able to account for the observations.

We are very grateful to the referee, Stuart Pottash for his comments that highly improve this paper. This work has been supported by NASA through grant 60-08271.01-97A from Space Telescope Science Institute, which is operated by the Association of Universities for Research in Astronomy.

\section{REFERENCES}

Bond, H. E. 2000, ASP Conf. Ser. 199: Asymmetrical Planetary Nebulae II: From Origins to Microstructures, 115

Cox, A. N. 2000, Allen's astrophysical quantities, 4th ed. Publisher: New York: AIP Press; Springer, 2000. Editedy by Arthur N. Cox. ISBN: 0387987460,

Allen, C. W. 1976, Astrophysical Quantities, (London: Athlone)

Bertelli, G., Mateo, M., Chiosi, C., \& Bressan, A. 1992, ApJ,

Boroson, T. A., \& Liebert, J. 1989, ApJ, 339, 844

Bowen, G. H. 1988, ApJ, 329, 299

Brown, T. et al. 2002, "HST STIS Data Handbook", version 4.0, ed. B. Mobasher, (Baltimore:STScI)

Chakravarti, Laha, \& Roy, (1967). Handbook of Methods of Applied Statistics, Volume I, John Wiley and Sons, pp. 392-394.

Code, A. D., Bless, R. C., Davis, J., \& Brown, R. H. 1976, ApJ, 203, 417 
Crowl, H. H., Sarajedini, A., Piatti, A. E., Geisler, D., Bica, E., Clariá, J. J., \& Santos, J. F. C. 2001, AJ, 122, 220

Dolphin, A. E., Walker, A. R., Hodge, P. W., Mateo, M., Olszewski, E. W., Schommer, R. A., \& Suntzeff, N. B. 2001, ApJ, 562, 303

Dominguez, I., Chieffi, A., Limongi, M., \& Straniero, O. 1999, ApJ, 524, 226

Dopita, M. A., Lawrence, C. J., Ford, H. C., \& Webster, B. L. 1985, ApJ, 296, 390

Dopita, M. A. \& Meatheringham, S. J. 1990, ApJ, 357, 140

Dopita, M. A. \& Meatheringham, S. J. 1991a, ApJ, 367, 115

Dopita, M. A. \& Meatheringham, S. J. 1991b, ApJ, 377, 480

Fasano, G. \& Franceschini, A. 1987, MNRAS, 225, 155

Gardiner, L. T. \& Hatzidimitriou, D. 1992, MNRAS, 257, 195

Gardiner, L. T. \& Hawkins, M. R. S. 1991, MNRAS, 251, 174

Gilliland, R. L., Goudfrooij, P. \& Kimble, R. A. 1999, PASP, 111, 1009

Girardi, L., Bressan, A., Bertelli, G., \& Chiosi, C. 2000, A\&AS, 141, 371

Gruenwald, R. \& Viegas, S. M. 2000, ApJ, 543, 889

Hardy, E., Suntzeff, N. B., \& Azzopardi, M. 1989, ApJ, 344, 210

Harman, R. F. \& Seaton, M. J. 1966, MNRAS, 132, 15

Hatzidimitriou, D. \& Hawkins, M. R. S. 1989, MNRAS, 241, 667

Hatzidimitriou, D., Hawkins, M. R. S., \& Gyldenkerne, K. 1989, MNRAS, 241, 645

Hatzidimitriou, D., Croke, B. F., Morgan, D. H., \& Cannon, R. D. 1997, A\&AS, 122, 507

Howarth, I. D. 1983, MNRAS, 203, 301

Iben, I. J. 1991, ApJS, 76, 55

Jacoby, G. H. 1981, ApJ, 244, 903

Jacoby, G. H., \& Kaler, J. B. 1993, ApJ, 417, 209

Kaler, J. B. 1983, ApJ, 271, 188 
Kaler, J. B. \& Jacoby, G. H. 1989, ApJ, 345, 871

Leisy, P. \& Dennefeld, M. 1996, A\&AS, 116, 95

Leisy, P. \& Dennefeld, M. 2003, A\&AS, in press

Leitherer et al.(2001) "STIS Instrument handbook", version 5.1, (Baltimore:STScI)

Monk, D. J., Barlow, M. J., \& Clegg, R. E. S. 1988, MNRAS, 234, 583

Olszewski, E. W., Suntzeff, N. B., \& Mateo, M. 1996, ARA\&A, 34, 511

Osterbrock, D. E. 1989, Astrophysics of Gaseous Nebulae and Active Galactic Nuclei (Mill Valley: University Science Books)

Peacock, J.A. 1983, MNRAS, 202, 615

Pottasch, S. R. 1984, Planetary Nebulae (Dordrecht: Reidel)

Pottasch, S. R. 1996, A\&A, 307, 561

Rejkuba, M., Minniti, D., Gregg, M. D., Zijlstra, A. A., Alonso, M. V. \& Goudfrooij, P., 2000, AJ, 120, 801

Renzini, A. \& Buzzoni, A. 1986, ASSL Vol. 122: Spectral Evolution of Galaxies, 195

Russell, S. C. \& Bessell, M. S. 1989, ApJS, 70, 865

Russell, S. C. \& Dopita, M. A. 1990, ApJS, 74, 93

Salpeter, E. E. 1955, ApJ, 121, 161

Seaton, M. J. 1979, MNRAS, 187, 73P

Savage, B. D. \& Mathis, J. S. 1979, ARA\&A 17, 73

Scalo, J. 1998, ASP Conf. Ser. 142: The Stellar Initial Mass Function (38th Herstmonceux Conference), 201

Stanghellini, L. 2000, Ap\&SS, 272, 181

Stanghellini, L., Shaw, R. A., Balick, B., \& Blades, J. C. 2000, ApJ, 534, L167

Stanghellini, L., Shaw, R. A., Mutchler, M., Palen, S., Balick, B., \& Blades, J. C. 2002, ApJ, 575, 178 
Stanghellini, L., Shaw, R. A., Balick, B., Mutchler, M., Balick, B., \& Blades, J. C., Villaver, E. 2003, ApJ, 596, 997

Stoy, R. H. 1933, MNRAS, 93, 588

Umeda, H., Nomoto, K., Yamaoka, H., \& Wanajo, S. 1999, ApJ, 513, 861

Vacca, W. D., Garmany, C. D., \& Shull, J. M. 1996, ApJ, 460, 914

Vassiliadis, E., Dopita, M. A., Morgan, D. H., \& Bell, J. F. 1992, ApJS, 83, 87

Vassiliadis, E.,\& Wood, P. R. 1994, ApJS, 92, 125

Vassiliadis, E. et al. 1998, ApJ, 503, 253

Villaver, E., García-Segura, G., \& Manchado, A. 2002a, ApJ, 571, 880

Villaver, E., Manchado, A., \& García-Segura, G. 2002b, ApJ, 581, 1204

Villaver, E., Stanghellini, L., \& Shaw, R. A. 2003, ApJ, 597, 298 (Paper I)

Westerlund, B. E., 1997, The Magellanic Clouds. Cambridge Univ. Press, Cambridge

Willson, L. A., Bowen, G. H., \& Struck, C. 1996, ASP Conf. Ser. 98: From Stars to Galaxies: the Impact of Stellar Physics on Galaxy Evolution, 197

Willson, L. A. 2000, ARA\&A, 38, 573

Winters, J. M., Le Bertre, T., Jeong, K. S., Helling, C., \& Sedlmayr, E. 2000, A\&A, 361, 641

Wood, P. R. 1979, ApJ, 227, 220

Yungelson, L. R., Tutukov, A. V., \& Livio, M. 1993, ApJ, 418, 794

Zanstra, H. 1931, Publ. Dom. Astrophys. OBs. Victoria, 4, 209 
Table 1. OBSERVATIONS

\begin{tabular}{lcc}
\hline \hline Name & $\begin{array}{c}\text { Integration } \\
\text { time }(\text { s })\end{array}$ & $\begin{array}{c}\text { CS } \\
\text { Detection } \\
(2)\end{array}$ \\
\hline J 4 & 300 & YES \\
J 18 & 300 & YES \\
J 23 & 300 & YES \\
J 27 & 300 & NO \\
MA 1682 & 300 & YES \\
MA 1762 & 300 & YES \\
MG 8 & 120 & YES \\
MG 13 & 300 & YES \\
SMP 1 & 120 & YES \\
SMP 6 & 120 & YES \\
SMP 8 & 120 & YES \\
SMP 9 & 300 & YES \\
SMP 11 & 120 & YES \\
SMP 12 & 300 & YES \\
SMP 13 & 120 & NO \\
SMP 14 & 120 & YES \\
SMP 17 & 120 & YES \\
SMP 18 & 120 & NO \\
SMP 19 & 120 & NO \\
SMP 20 & 120 & YES \\
SMP 22 & 120 & NO \\
SMP 23 & 120 & YES \\
SMP 24 & 120 & YES \\
SMP 25 & 120 & YES \\
SMP 26 & 300 & NO \\
SMP 27 & 120 & YES \\
SP 34 & 300 & NO \\
\hline
\end{tabular}


Table 2. MAGNitudes, EXTINCTION, AND He il FLUXES

\begin{tabular}{|c|c|c|c|c|c|}
\hline $\begin{array}{c}\text { Name } \\
(1)\end{array}$ & $\begin{array}{c}\text { STMAG } \\
(2)\end{array}$ & $\begin{array}{l}\mathrm{V} \\
(3)\end{array}$ & $\begin{array}{c}\mathrm{c} \\
(4)\end{array}$ & $\begin{array}{c}\mathrm{I}(\mathrm{He} \mathrm{II}) \\
(5)\end{array}$ & $\begin{array}{c}\text { Reference } \\
(6)\end{array}$ \\
\hline $\mathrm{J} 4^{\mathrm{a}}$ & $19.10 \pm 0.03$ & $18.81 \pm 0.07$ & 0.17 & $73.0 \pm 7.3$ & $\mathrm{BL}$ \\
\hline J 18 & $20.93 \pm 0.04$ & $19.89 \pm 0.06$ & 0.42 & $20.40 \pm 4.0$ & JK93 \\
\hline J 23 & $20.17 \pm 0.04$ & $16.71 \pm 0.05$ & 1.65 & $57.0 \pm 5.7$ & $\mathrm{BL}$ \\
\hline J 27 & $\geq 26.89$ & $\geq 25.89$ & 0.41 & $9.0 \pm 2.1$ & $\mathrm{BL}$ \\
\hline MA 1682 & $24.00 \pm 0.05$ & $24.30 \pm 0.10^{\mathrm{b}}$ & $\ldots$ & $\ldots$ & $\ldots$ \\
\hline MA 1762 & $23.86 \pm 0.08$ & $24.17 \pm 0.13^{\mathrm{b}}$ & $\ldots$ & $\ldots$ & $\ldots$ \\
\hline MG 8 & $18.26 \pm 0.02$ & $18.09 \pm 0.06$ & 0.13 & $28.5 \pm 1.4$ & LEI1 \\
\hline MG 13 & $21.67 \pm 0.02$ & $21.03 \pm 0.05$ & 0.28 & $97.5 \pm 5.0$ & LEI1 \\
\hline SMP 1 & $17.65 \pm 0.02$ & $16.99 \pm 0.05$ & 0.29 & $\leq 1.0$ & MEA \\
\hline SMP 6 & $17.66 \pm 0.12$ & $16.71 \pm 0.14$ & 0.39 & $\leq 0.5$ & LEI \\
\hline SMP 8 & $18.06 \pm 0.05$ & $18.27 \pm 0.10$ & 0.03 & 0.0 & MON \\
\hline SMP 9 & $24.37 \pm 0.41$ & $24.41 \pm 0.45$ & 0.07 & $59.6 \pm 3.0$ & LEI \\
\hline SMP 11 & $20.25 \pm 0.14$ & $19.40 \pm 0.16$ & 0.35 & $\leq 1.0$ & LEI1 \\
\hline SMP 12 & $19.70 \pm 0.04$ & $19.80 \pm 0.08$ & 0.06 & $\leq 2.0$ & LEI1 \\
\hline SMP 13 & $\geq 17.17$ & $\geq 16.87$ & 0.19 & $\leq 0.3$ & LEI \\
\hline SMP 14 & $21.69 \pm 0.28$ & $21.74 \pm 0.32$ & 0.07 & $37.8 \pm 1$. & LEI \\
\hline SMP 17 & $19.34 \pm 0.15$ & $19.41 \pm 0.19$ & 0.06 & $\leq 1.0$ & VAS \\
\hline $\mathrm{SMP} 18^{\mathrm{a}}$ & $17.15 \pm 0.05$ & $17.06 \pm 0.09$ & 0.12 & 0.0 & MON \\
\hline SMP 19 & $\geq 20.79$ & $\geq 20.49$ & 0.16 & $40.0 \pm 1.2$ & LEI \\
\hline SMP 20 & $20.30 \pm 0.02$ & $20 . \overline{42} \pm 0.07$ & 0.05 & $\leq 0.3$ & LEI \\
\hline SMP 22 & $\geq 21.04$ & $\geq 20.73$ & 0.17 & $60 . \overline{4} \pm 1.2$ & LEI \\
\hline SMP 23 & $19 . \overline{74} \pm 0.09$ & $19 . \overline{68} \pm 0.13$ & 0.10 & $2.8 \pm 0.1$ & LEI \\
\hline SMP 24 & $17.85 \pm 0.03$ & $17.98 \pm 0.07$ & 0.05 & 0.0 & MON \\
\hline SMP 25 & $18.65 \pm 0.01$ & $18.59 \pm 0.05$ & 0.10 & $53.3 \pm 3.2$ & LEI \\
\hline SMP 26 & $\geq 22.65$ & $\geq 22.06$ & 0.25 & $52.5 \pm 2.6$ & LEI1 \\
\hline SMP 27 & $18.04 \pm 0.04$ & $18.20 \pm 0.09$ & 0.04 & 0.0 & MON \\
\hline SP 34 & $\geq 23.08$ & $\geq 22.77$ & 0.16 & $\ldots$ & $\ldots$ \\
\hline
\end{tabular}

Note. $-1-\sigma$ errors are quoted throughout. The $\geq$ symbol refers to lower limit to the magnitude when the CS is not detected.

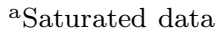

${ }^{\mathrm{b}}$ No extinction constant available. The $\mathrm{V}$ mag was computed assuming zero extinction

References. - (BL)Boroson \& Liebert (1989); (JK93)Jacoby \& Kaler (1993);(LEI1) Leisy \& Dennefeld (2003); (MEA) Dopita \& Meatheringham (1991a,b); (LEI) Leisy \& Dennefeld (1996); (VAS) Vassiliadis et al. (1992); (MON) Monk, Barlow, \& Clegg (1988) 
Table 3. CS PARAMETERS

\begin{tabular}{|c|c|c|c|c|c|c|c|}
\hline $\begin{array}{l}\text { NAME } \\
(1)\end{array}$ & $\begin{array}{c}\mathrm{T}_{\mathrm{eff}}\left(\mathrm{He}_{I I}\right) \\
\left(10^{3} \mathrm{~K}\right) \\
(2)\end{array}$ & $\begin{array}{c}\mathrm{T}_{\mathrm{eff}}(\mathrm{H}) \\
\left(10^{3} \mathrm{~K}\right) \\
(3)\end{array}$ & $\begin{array}{r}\mathrm{M}_{\mathrm{V}} \\
(4)\end{array}$ & $\begin{array}{c}\log L_{*} / L_{\odot} \\
(5)\end{array}$ & $\begin{array}{l}\mathrm{BC} \\
(6)\end{array}$ & $\begin{array}{l}\mathrm{M} \\
(7)\end{array}$ & $\begin{array}{c}\text { COMMENTS } \\
(8)\end{array}$ \\
\hline $\mathrm{J} 4$ & $74.1 \pm 5.1$ & $29.0 \pm 3.2$ & $-0.02 \pm 0.07$ & $4.17 \pm 0.09$ & $-5.65 \pm 0.20$ & $\mathrm{E}$ & Saturated \\
\hline J 18 & $52.6 \pm 2.3$ & $20.2 \pm 1.3$ & $1.06 \pm 0.06$ & $3.33 \pm 0.06$ & $-4.63 \pm 0.13$ & $\mathrm{R}$ & \\
\hline J 23 & $54.9 \pm 2.8$ & $18.6 \pm 1.4$ & $-2.12 \pm 0.05$ & $4.65 \pm 0.06$ & $-4.76 \pm 0.15$ & $\mathrm{U}$ & Possibly not a PN \\
\hline $\mathrm{J} 27$ & $91.3 \pm 7.2$ & $78.9 \pm 14.7$ & $\geq 7.03$ & $\leq 1.63$ & $-6.27 \pm 0.23$ & B & Very faint object \\
\hline MA 1682 & $\cdots$ & $\cdots$ & $5.47 \pm 0.1$ & $\cdots$ & $\cdots$ & B & no $\mathrm{H} \beta$ fluxes, nor c available \\
\hline MA 1762 & $\cdots$ & $\cdots$ & $5.34 \pm 0.13$ & $\cdots$ & $\cdots$ & $\mathrm{E}(\mathrm{bc})$ & no $\mathrm{H} \beta$ fluxes, nor c available \\
\hline MG 8 & $66.5 \pm 3.3$ & $28.8 \pm 2.6$ & $-0.74 \pm 0.06$ & $4.33 \pm 0.06$ & $-5.33 \pm 0.15$ & $\mathrm{E}$ & \\
\hline MG 13 & $98.3 \pm 8.7$ & $43.7 \pm 6.8$ & $2.20 \pm 0.05$ & $3.62 \pm 0.11$ & $-6.49 \pm 0.26$ & $\mathrm{E}$ & \\
\hline SMP 1 & $\leq 46.4^{\mathrm{a}}$ & $28.8 \pm 2.6$ & $-1.84 \pm 0.05$ & $3.77 \pm 0.11$ & $-2.84 \pm 0.27$ & $\mathrm{U}$ & \\
\hline SMP 6 & $\leq 43.7^{\mathrm{a}}$ & $28.2 \pm 2.5$ & $-2.11 \pm 0.14$ & $3.86 \pm 0.12$ & $-2.78 \pm 0.26$ & $\mathrm{E}$ & \\
\hline SMP 8 & $\cdots$ & $37.1 \pm 4.1$ & $-0.56 \pm 0.10$ & $3.56 \pm 0.14$ & $-3.59 \pm 0.33$ & $\mathrm{R}$ & \\
\hline SMP 9 & $175.6 \pm 27.2$ & $156.8 \pm 44.8$ & $5.59 \pm 0.45$ & $2.95 \pm 0.26$ & $-8.21 \pm 0.46$ & $\mathrm{R}$ & Photometry in Nebula subs image \\
\hline SMP 11 & $\leq 52.6^{\mathrm{a}}$ & $40.9 \pm 5.0$ & $0.57 \pm 0.16$ & $3.22 \pm 0.16$ & $-3.88 \pm 0.36$ & $\mathrm{~B}$ & Photometry in Nebula subs image \\
\hline SMP 12 & $\leq 51.7^{\mathrm{a}}$ & $34.0 \pm 4.4$ & $0.97 \pm 0.08$ & $2.84 \pm 0.16$ & $-3.34 \pm 0.38$ & $\mathrm{E}$ & \\
\hline SMP 13 & $\leq 44.0^{\mathrm{a}}$ & $31.3 \pm 2.2$ & $\geq-2.05$ & $\leq 4.03$ & $-3.09 \pm 0.21$ & $\mathrm{R}$ & \\
\hline SMP 14 & $116.8 \pm 11.4$ & $83.5 \pm 17.6$ & $2.92 \pm 0.32$ & $3.53 \pm 0.17$ & $-7.00 \pm 0.29$ & $\mathrm{R}$ & \\
\hline SMP 17 & $\leq 58.9^{\mathrm{a}}$ & $58.4 \pm 7.3$ & $0.58 \pm 0.19$ & $3.65 \pm 0.17$ & $-4.94 \pm 0.37$ & $\mathrm{E}$ & Photometry in Nebula subs image \\
\hline SMP 18 & $\cdots$ & $31.5 \pm 2.3$ & $-1.76 \pm 0.09$ & $3.85 \pm 0.09$ & $-3.11 \pm 0.22$ & $\mathrm{U}$ & Saturated \\
\hline SMP 19 & $100.6 \pm 7.7$ & $59.4 \pm 9.4$ & $\geq 1.66$ & $\leq 3.90$ & $-6.56 \pm 0.23$ & $\mathrm{R}$ & \\
\hline SMP 20 & $\leq 58.5^{\mathrm{a}}$ & $86.5 \pm 11.8$ & $1.59 \pm 0.07$ & $3.71 \pm 0.16$ & $-6.11 \pm 0.40$ & $\mathrm{U}$ & \\
\hline SMP 22 & $122.2 \pm 14.5$ & $76.7 \pm 18.2$ & $\geq 1.91$ & $\leq 4.04$ & $-7.14 \pm 0.35$ & $\mathrm{~B}$ & \\
\hline SMP 23 & $62.1 \pm 3.0$ & $41.6 \pm 5.1$ & $0 . \overline{85} \pm 0.13$ & $3 . \overline{6} 1 \pm 0.08$ & $-5.12 \pm 0.14$ & $\mathrm{E}(\mathrm{bc})$ & \\
\hline SMP 24 & $\cdots$ & $37.8 \pm 3.2$ & $-0.85 \pm 0.07$ & $3.70 \pm 0.10$ & $-3.65 \pm 0.25$ & $\mathrm{E}$ & \\
\hline SMP 25 & $74.8 \pm 4.1$ & $31.4 \pm 3.0$ & $-0.23 \pm 0.05$ & $4.26 \pm 0.07$ & $-5.68 \pm 0.16$ & $\mathrm{E}$ & \\
\hline SMP 26 & $111.6 \pm 12.3$ & $67.0 \pm 15.0$ & $\geq 3.23$ & $\leq 3.41$ & $-6.87 \pm 0.33$ & $\mathrm{P}$ & \\
\hline SMP 27 & $\ldots$ & $43.3 \pm 4.1$ & $-0.63 \pm 0.09$ & $3.77 \pm 0.12$ & $-4.05 \pm 0.28$ & $\mathrm{R}$ & \\
\hline SP 34 & $\cdots$ & $80.3 \pm 20.9$ & $\geq 3.94$ & $\leq 2.87$ & $-5.89 \pm 0.77$ & $\mathrm{R}$ & \\
\hline
\end{tabular}

Note. $-1-\sigma$ errors are quoted throughout. The $\geq$ symbol refers to the lower limit to the magnitude when the CS is not detected. In which case, the luminosities are upper limits and are preceded by a $\leq$ symbol.

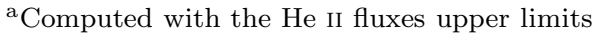




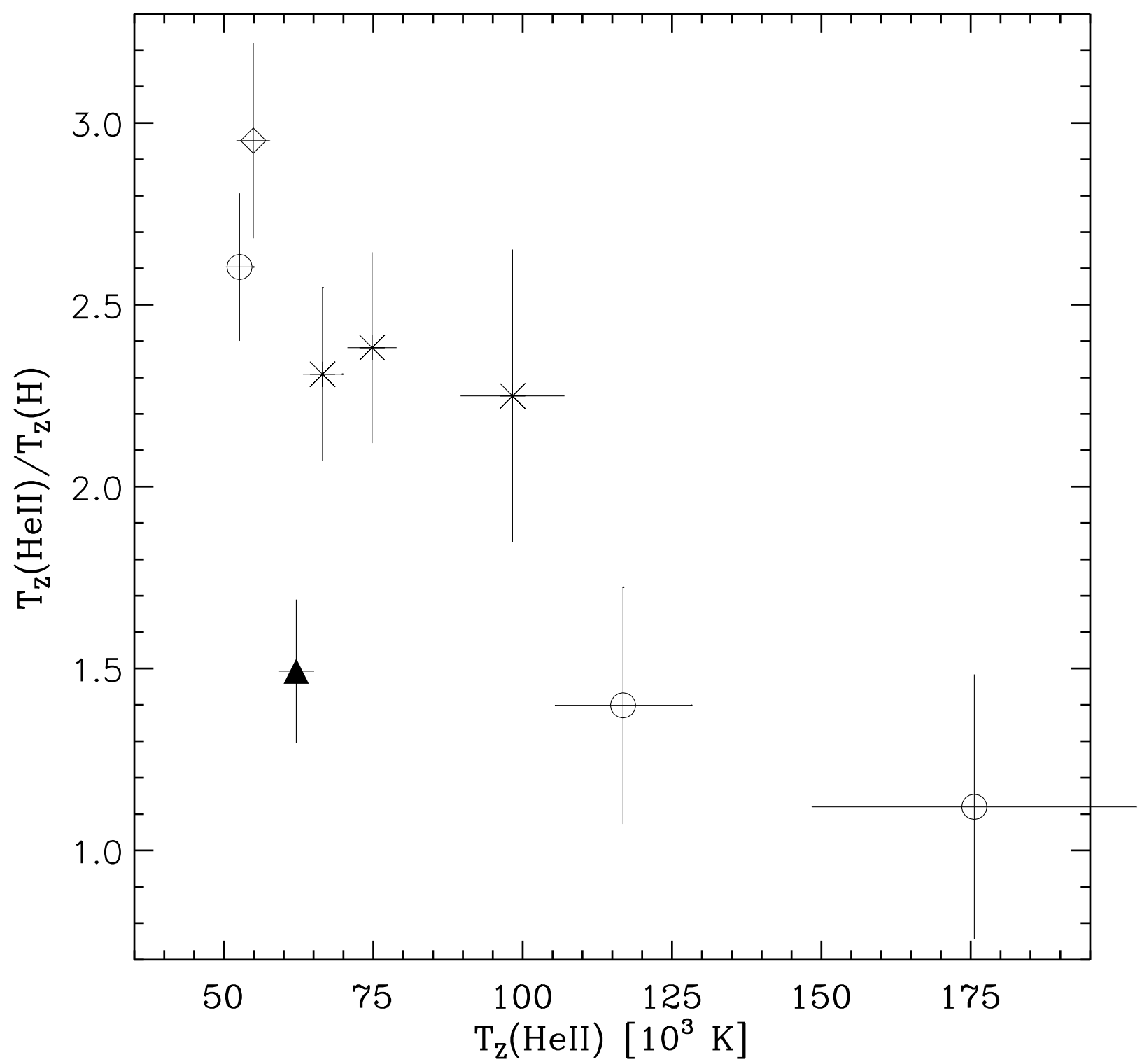

Fig. 1.- Zanstra ratio versus He II Zanstra temperature. The symbols represent the morphological types of the hosting nebulae: round (open circles), elliptical (asterisks), bipolar and quadrupolar (squares), bipolar core (triangles), point-symmetric (filled circles) and unresolved (diamonds). 


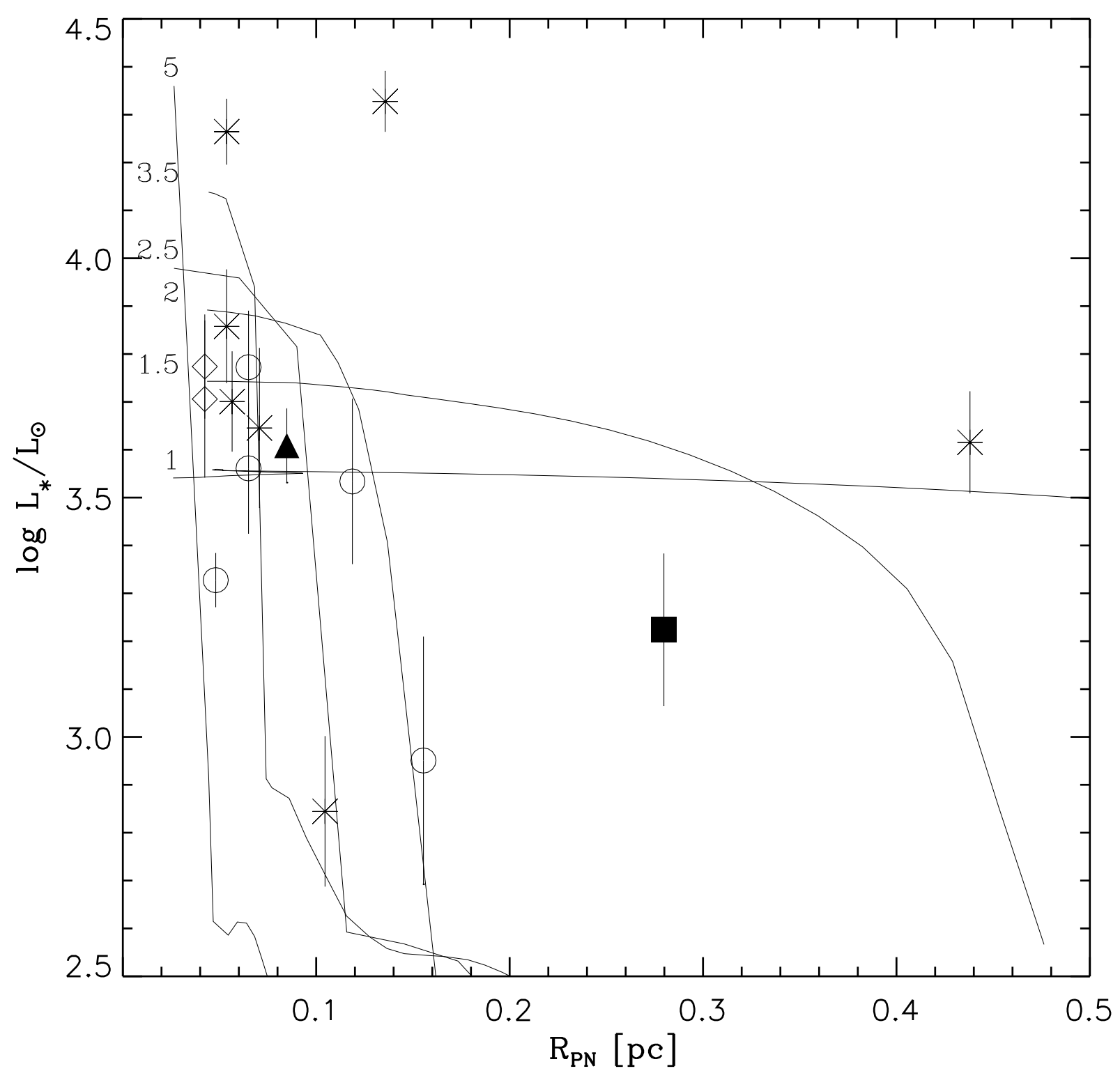

Fig. 2.- The points represent the logarithm of the observed luminosity versus the physical radius of the nebulae. The symbols are the same as used in Fig. 1. The solid line represent the evolution of the nebular radius versus the stellar luminosity taken from the numerical simulations of Villaver, Manchado, \& García-Segura (2002b) for Galactic PN. Each line has been marked with the initial mass of the progenitor used in the hydrodynamical simulation. 


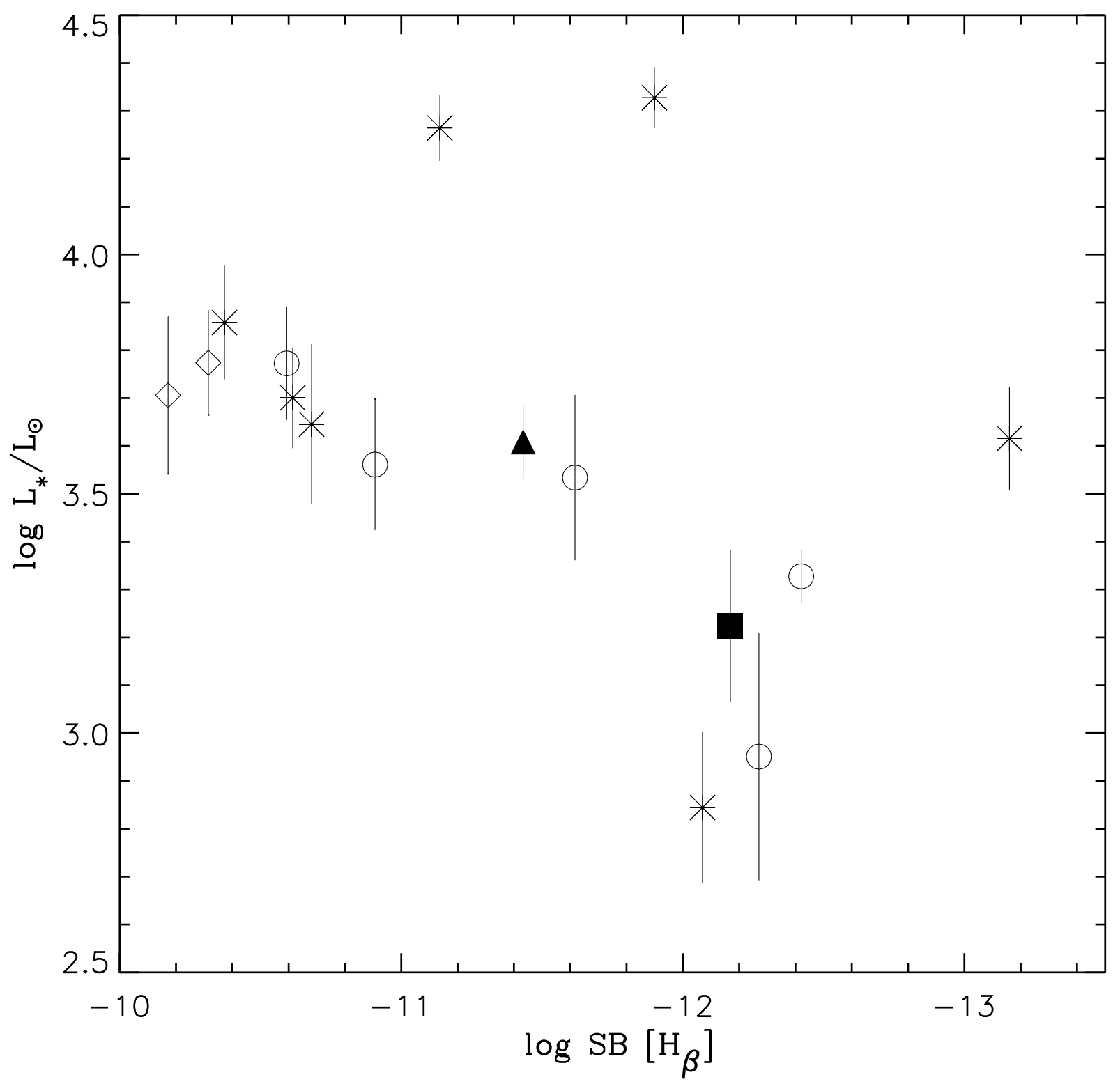

Fig. 3.- Logarithm of the observed luminosity versus the surface brightness of the nebula in the $\mathrm{H} \beta$ lines. The symbols are the same as used in Fig. 1. 


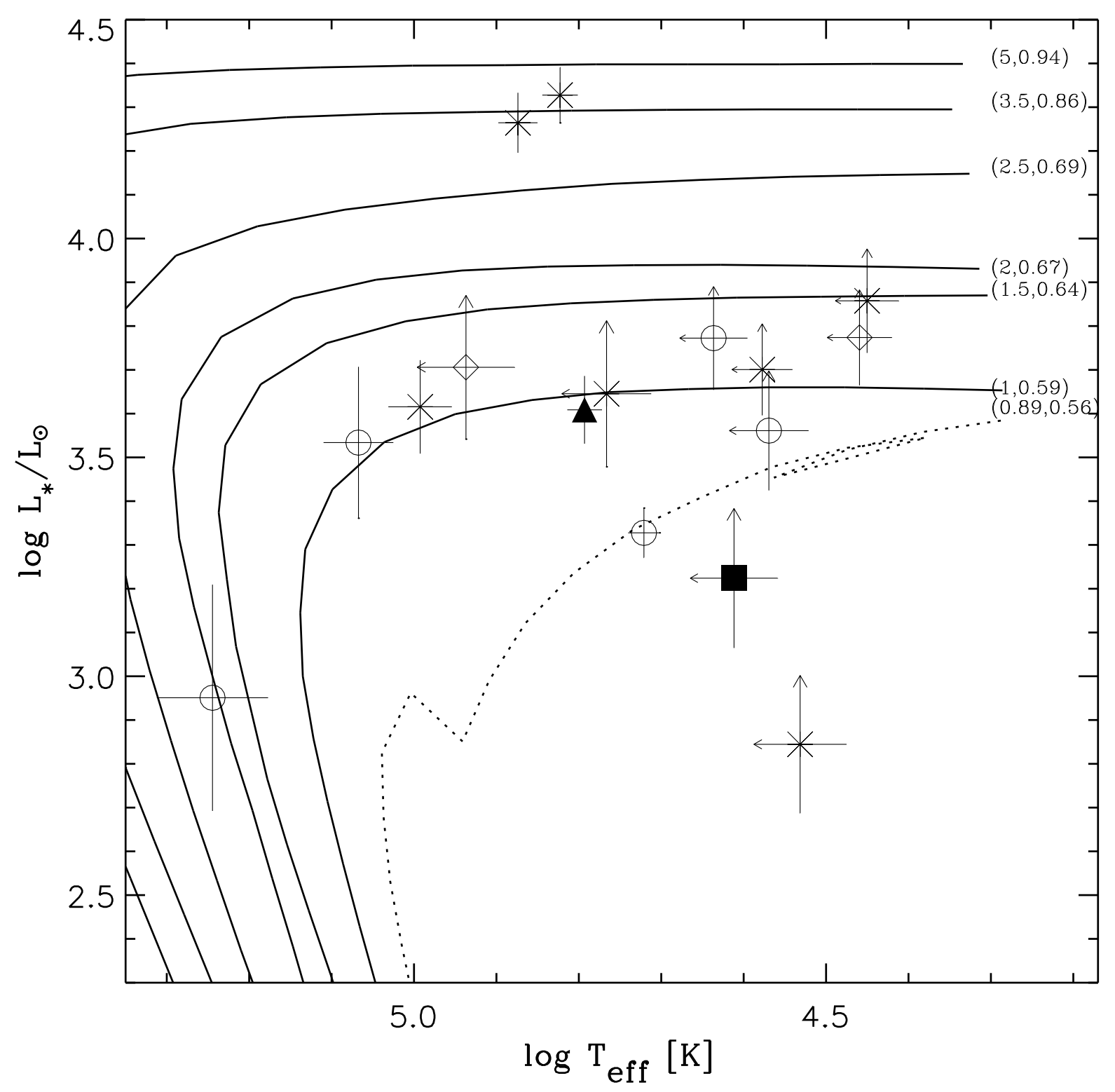

Fig. 4.- HR diagram for the CSs. Symbols are the same as used in Fig. 1. We have maked with arrows those points for which we have used the H I Zanstra temperatures. Evolutionary tracks for SMC metallicities are from Vassiliadis \& Wood (1994). The initial and final masses are marked on each track. The dotted lines is for a He-burnering track and the solid lines for H-burners. 


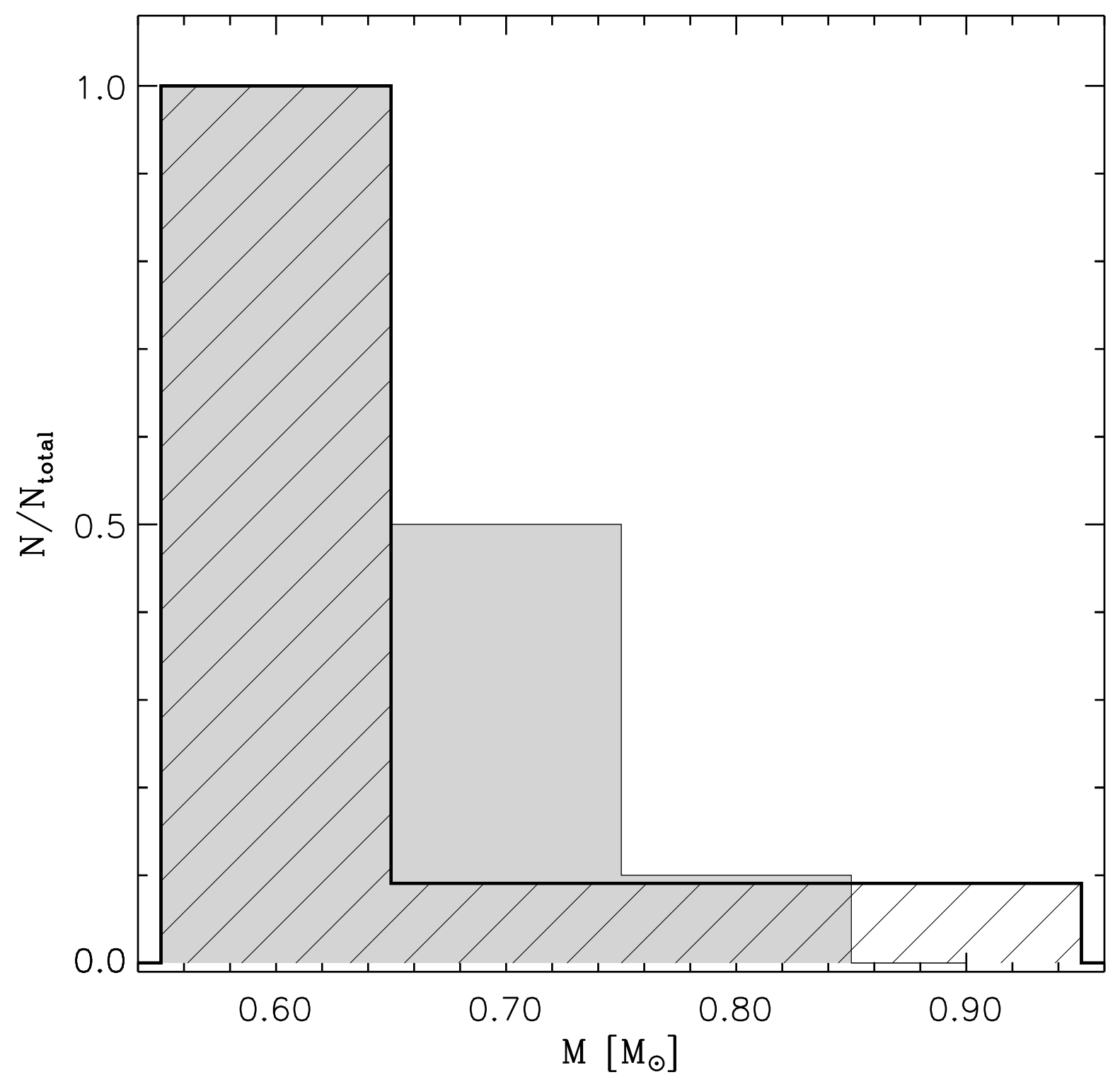

Fig. 5.- Normalized mass distribution for the CSs in the SMC (dashed) and LMC (in gray). 


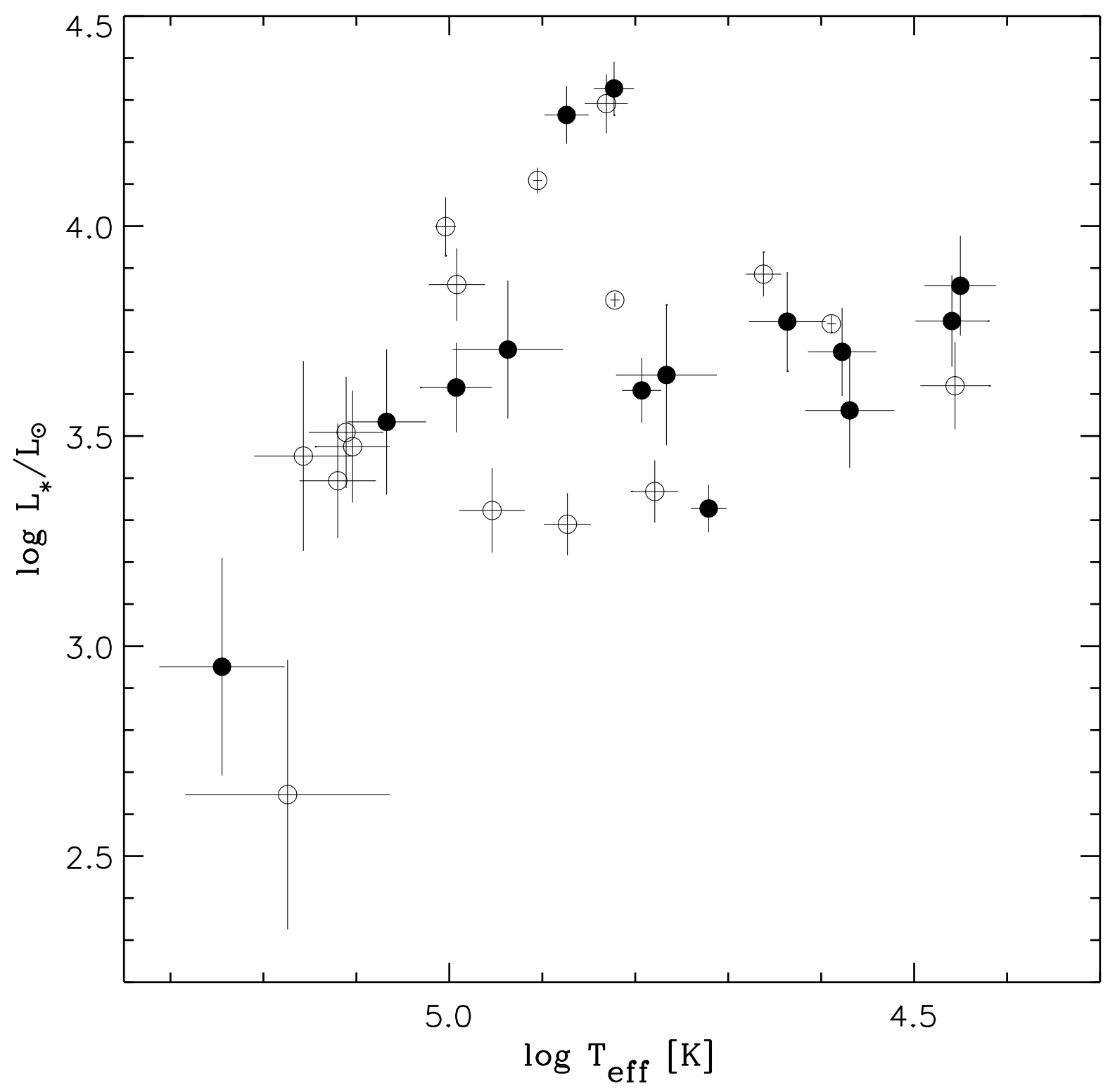

Fig. 6.- HR diagram for the CSs in the SMC (filled circles) and LMC (open circles). Only those points for which the mass has been determined is plotted. 
Table 4. MASSES OF THE CENTRAL STARS

\begin{tabular}{lcc}
\hline \hline Name & M $\left[\mathrm{M}_{\odot}\right]$ & Comments \\
\hline J 18 & 0.56 & He-burning track \\
MG 8 & 0.88 & Core mass-Luminosity relation \\
MG 13 & 0.59 & Extrapolation H-burning track \\
SMP 1 & $0.60^{\mathrm{a}}$ & Core mass-Luminosity relation \\
SMP 6 & $0.63^{\mathrm{a}}$ & Core mass-Luminosity relation \\
SMP 8 & $0.56^{\mathrm{a}}$ & Core mass-Luminosity relation \\
SMP 9 & 0.67 & H-burning track \\
SMP 14 & 0.59 & H-burning track \\
SMP 17 & $0.59^{\mathrm{a}}$ & H-burning track \\
SMP 20 & $0.59^{\mathrm{a}}$ & Core mass-Luminosity relation \\
SMP 23 & 0.59 & Extrapolation H-burning track \\
SMP 24 & $0.59^{\mathrm{a}}$ & Core mass-Luminosity relation \\
SMP 25 & 0.82 & Core mass-Luminosity relation \\
SMP 27 & $0.60^{\text {a }}$ & Core mass-Luminosity relation \\
\hline
\end{tabular}

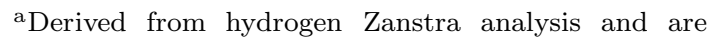
therefore rather uncertain (see text). Note that the mass derived from the He-burning track might be slightly lower than the mass derived from a H-burning track. 
Table 5. BINARY COMPANION TO THE CS

\begin{tabular}{|c|c|c|c|c|c|c|c|c|c|}
\hline $\begin{array}{l}\text { Name } \\
(1)\end{array}$ & $\begin{array}{l}\text { \% contribution } \\
(2)\end{array}$ & $\begin{array}{l}\text { mpanio } \\
\qquad \\
(3)\end{array}$ & $\begin{array}{l}\text { Star } \\
\text { Sp type } \\
\quad(4)\end{array}$ & $\begin{array}{c}\mathrm{M}\left[\mathrm{M}_{\odot}\right] \\
(5)\end{array}$ & $\begin{array}{l}V \\
(6)\end{array}$ & $\begin{array}{c}\mathrm{T}_{\mathrm{eff}}\left(10^{3} \mathrm{~K}\right) \\
\quad(7)\end{array}$ & $\begin{array}{c}\text { Central Sta } \\
\log L_{*} / L_{\odot} \\
\quad(8)\end{array}$ & $\begin{array}{c}\mathrm{M}_{C}\left[\mathrm{M}_{\odot}\right] \\
\quad(9)\end{array}$ & $\begin{array}{c}\mathrm{M}_{i}\left[\mathrm{M}_{\odot}\right] \\
\quad(10)\end{array}$ \\
\hline \multirow[t]{3}{*}{ J 18} & 50 & 20.10 & $\mathrm{~A} 2$ & 2.5 & 20.65 & 55.9 & 3.09 & $\mathrm{a}$ & $\ldots$ \\
\hline & 90 & 19.46 & $\mathrm{~A} 1$ & 2.7 & 22.44 & 65.5 & 2.57 & $\mathrm{a}$ & $\ldots$ \\
\hline & 95 & 19.40 & $\mathrm{~A} 1$ & 2.7 & 23.27 & 71.1 & 2.33 & $\mathrm{a}$ & $\ldots$ \\
\hline \multirow[t]{3}{*}{ MG 08} & 50 & 18.57 & B8 & 3.0 & 19.02 & 73.1 & 4.06 & 0.69 & 2.5 \\
\hline & 90 & 17.93 & B7 & 3.0 & 20.77 & 89.7 & 3.61 & 0.59 & 1 \\
\hline & 95 & 17.87 & B7 & 3.0 & 21.52 & 99.2 & 3.42 & 0.57 & 0.9 \\
\hline \multirow[t]{3}{*}{ MG 13} & 50 & 21.17 & $\mathrm{~A} 7$ & 1.8 & 21.78 & 109.8 & 3.45 & 0.59 & 1 \\
\hline & 90 & 20.53 & A 7 & 1.8 & 23.51 & 148.8 & 3.11 & 0.61 & 1.2 \\
\hline & 95 & 20.47 & $\mathrm{~A} 7$ & 1.8 & 24.36 & 178.7 & 2.99 & 0.67 & 2 \\
\hline \multirow[t]{3}{*}{ SMP 1} & 50 & 17.55 & B3 & 7.6 & 17.75 & 33.2 & 3.64 & 0.59 & 1 \\
\hline & 90 & 16.92 & B3 & 7.6 & 19.44 & 48.9 & 3.42 & 0.56 & 0.89 \\
\hline & 95 & 16.89 & B3 & 7.6 & 19.70 & 52.4 & 3.40 & 0.56 & 0.89 \\
\hline \multirow[t]{3}{*}{ SMP 6} & 50 & 17.28 & B3 & 7.6 & 17.46 & 32.4 & 3.72 & 0.59 & 1 \\
\hline & 90 & 16.64 & B3 & 7.6 & 19.21 & 48.0 & 3.49 & $\sim 0.57$ & $\sim 0.9$ \\
\hline & 95 & 16.58 & B3 & 7.6 & 19.99 & 59.6 & 3.44 & $\sim 0.57$ & $\sim 0.9$ \\
\hline \multirow[t]{3}{*}{ SMP 8} & 50 & 18.68 & B7 & 7.6 & 19.03 & 44.2 & 3.47 & 0.56 & 0.89 \\
\hline & 90 & 18.05 & B6 & 7.6 & 20.73 & 71.7 & 3.36 & $\sim 0.57$ & $\sim 0.9$ \\
\hline & 95 & 17.99 & B6 & 7.6 & 21.46 & 92.4 & 3.37 & $\sim 0.57$ & $\sim 0.9$ \\
\hline \multirow[t]{3}{*}{ SMP 9} & 50 & 24.60 & K0 & 0.79 & 25.16 & 210.9 & 2.87 & 0.70 & 2.5 \\
\hline & 90 & 23.96 & G8 & 0.85 & 26.91 & 363.9 & 2.82 & $\mathrm{a}$ & $\ldots$ \\
\hline & 95 & 23.90 & G8 & 0.85 & 27.68 & 501.0 & 2.89 & $\mathrm{a}$ & $\ldots$ \\
\hline \multirow[t]{3}{*}{ SMP 14} & 50 & 21.89 & F0 & 1.6 & 22.50 & 133.2 & 3.39 & 0.59 & 1 \\
\hline & 90 & 21.25 & F0 & 1.6 & 24.29 & 194.7 & 3.12 & 0.67 & 2 \\
\hline & 95 & 21.20 & F0 & 1.6 & 24.92 & 229.5 & 3.06 & 0.70 & 2.5 \\
\hline \multirow[t]{3}{*}{ SMP 17} & 50 & 19.61 & $\mathrm{~A} 0$ & 2.9 & 20.17 & 74.1 & 3.62 & 0.59 & 1 \\
\hline & 90 & 18.97 & $\mathrm{~A} 0$ & 2.9 & 21.95 & 144.9 & 3.71 & 0.64 & 1.5 \\
\hline & 95 & 18.92 & $\mathrm{~A} 0$ & 2.9 & 22.59 & 190.1 & 3.77 & 0.68 & 2.2 \\
\hline \multirow[t]{3}{*}{ SMP 20} & 50 & 20.57 & A4 & 2.3 & 21.17 & 114.5 & 3.74 & 0.64 & 1.5 \\
\hline & 90 & 19.93 & $\mathrm{~A} 3$ & 2.3 & 22.89 & 236.4 & 3.91 & 0.69 & 2.5 \\
\hline & 95 & 19.87 & A3 & 2.3 & 23.64 & 331.0 & 4.01 & 0.86 & 3.5 \\
\hline \multirow[t]{3}{*}{ SMP 23} & 50 & 19.87 & $\mathrm{~A} 0$ & 2.9 & 20.44 & 66.8 & 3.39 & $\sim 0.57$ & $\sim 0.89$ \\
\hline & 90 & 19.24 & A0 & 2.9 & 22.14 & 80.0 & 2.93 & 0.56 & 0.89 \\
\hline & 95 & 19.18 & A0 & 2.9 & 22.88 & 87.4 & 2.74 & 0.56 & 0.89 \\
\hline \multirow[t]{3}{*}{ SMP 24} & 50 & 18.39 & B6 & 5.2 & 18.73 & 45.1 & 3.61 & 0.59 & 1 \\
\hline & 90 & 17.75 & B6 & 5.2 & 20.50 & 75.4 & 3.51 & $\sim 0.58$ & $\sim 1$ \\
\hline & 95 & 17.69 & B6 & 5.2 & 21.29 & 100.0 & 3.53 & 0.59 & 1 \\
\hline \multirow[t]{3}{*}{ SMP 25} & 50 & 18.87 & B8 & 3.8 & 19.35 & 81.5 & 4.06 & 0.69 & 2.5 \\
\hline & 90 & 18.24 & B8 & 3.8 & 21.06 & 101.8 & 3.64 & 0.61 & 1.2 \\
\hline & 95 & 18.18 & B8 & 3.8 & 21.79 & 113.7 & 3.48 & 0.59 & 1 \\
\hline \multirow[t]{3}{*}{ SMP 27} & 50 & 18.58 & B7 & 4.5 & 18.96 & 52.8 & 3.70 & 0.60 & 1.1 \\
\hline & 90 & 17.95 & B7 & 4.5 & 20.66 & 90.9 & 3.67 & 0.60 & 1.1 \\
\hline & 95 & 17.89 & B7 & 4.5 & 21.39 & 119.9 & 3.71 & 0.63 & 1.5 \\
\hline
\end{tabular}

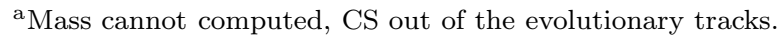


Table 5. STATISTICS

\begin{tabular}{ccccccc}
\hline \hline & \multicolumn{2}{c}{ Mass $\left[\mathrm{M}_{\odot}\right]$} & \multicolumn{2}{c}{$\log T_{\text {eff }}[\mathrm{K}]$} & \multicolumn{2}{c}{$\log L_{*} / L_{\odot}$} \\
galaxy & $<M>$ & Median & $<\log T_{\text {eff }}>$ & Median & $<\log L_{*} / L_{\odot}>$ & Median \\
$(1)$ & $(2)$ & $(3)$ & $(4)$ & $(5)$ & $(6)$ & $(7)$ \\
\hline SMC(14) & 0.63 & 0.59 & $4.78 \pm 0.23$ & 4.79 & $3.69 \pm 0.34$ & 3.70 \\
LMC(16) & 0.65 & 0.63 & $4.91 \pm 0.21$ & 4.95 & $3.61 \pm 0.40$ & 3.62 \\
\hline
\end{tabular}

Note. $-1-\sigma$ errors are given. 